\title{
Using Telemedicine for Outpatient Geriatric Care During the Novel Coronavirus Outbreak: Experience from the First 15 Patients
}

\section{Dear Editor,}

The coronavirus disease 2019 (COVID-19) outbreak first began in early December in Wuhan, China and was reported by World Health Organisation on 31 st December 2019. ${ }^{1}$ The outbreak escalated and was characterised as a pandemic on 11 March 2020. ${ }^{1}$ Amidst the avalanche of COVID-19 cases, Singapore's Ministry of Health implemented a series of Circuit Breaker (CB) measures on 7 April 2020, whereby non-essential specialist appointments were advised to be deferred and where possible, for services to continue via teleconsultation. ${ }^{2}$ The CB measures were in place until 1 June 2020, and as of 30 June 2020, Singapore had a total of 43,635 cases and 26 deaths. ${ }^{3}$

While specialist geriatric care does not constitute urgent care, many geriatric issues can lead to complications if not addressed on a timely basis. Uncontrolled geriatric problems, particularly behavioural and psychological symptoms in dementia (BPSD) is known to lead to further medical complications, which inevitably worsen caregivers' well-being. ${ }^{4}$ Furthermore, geriatric patients often present with more co-morbidities and non-specific presenting symptoms, which lead to transfers to emergency department, if not promptly recognised and managed. ${ }^{5}$ Prevention of hospitalisations for this vulnerable group during the pandemic is critical. Therefore, it is imperative to ensure that geriatric patients are able to access specialist geriatric care in the midst of a pandemic.

Telemedicine is routinely used for geri-psychiatric consultations and has been acknowledged as a useful tool in combating epidemics. ${ }^{6,7}$ In the current global outbreak, where social distancing is key in slowing the transmission of COVID-19 virus, telemedicine via videoconference is ideal in providing remote assessment, routine care and improved provider diagnostic accuracy compared to phone consults, without the risk of exposure in a congested hospital or in clinic waiting areas. ${ }^{1,3,8,9}$

In view of the above circumstances, a pilot Telemedicine Specialist Outpatient Clinic (Tele-SOC) unit was set up within the Geriatric Medicine clinic in an acute hospital in Singapore. In this paper, we shared a proof of concept study, which comprised of the demographics of the first 15 patients, protocol and feedback from patients, caregivers and the healthcare professionals (HCPs) involved.

\section{Description of Tele-SOC}

\section{Equipment}

A video conferencing application for healthcare, which attained Health Insurance Portability and Accountability Act (signed Business associate agreement) and Personal Information Protection and Electronic Documents Act/ Personal Health Information Protection Act compliance with complete end-toend 256-bit Advanced Encryption Standard (AES) encryption was used for Tele-SOC consultations. ${ }^{10}$ At the hospital's Tele-SOC unit, 2 laptops with Intel Core i5 processors were used with the laptops' inbuilt webcam, speaker and microphone. Patients and/ or caregivers were instructed to download the video conferencing application on to their smartphones or laptops with the help of written instructions. ${ }^{10}$

\section{Criteria and Protocol}

Figure 1 presents the entry and exclusion criteria, as well as the protocol for Tele-SOC. Suitable patients were offered the option of a teleconsultation with a geriatrician in lieu of a physical visit. All stakeholders were aware that privacy, confidentiality, communication, documentation and safety standards had to be strictly adhered to.

\section{Manpower}

The Tele-SOC unit was run by 1 geriatrician and assisted by a geriatric nurse, who were trained to set up the system and to troubleshoot technical problems associated with the videoconference application. ${ }^{10}$ A total of 2 geriatricians and 4 nurses used this platform on a rotational basis.

\section{Methods}

We collected demographics and diagnoses of the first 15 patients who were seen by Tele-SOC over a 2 week period. Feedback was given by the patients or their caregivers (if patients were unable to provide due to a lack of mental capacity) via phone calls using a structured interview format based on a questionnaire created by Tele- 




Fig. 1. Criteria and Protocol for Tele-SOC. 
SOC. The same set of questionnaires with variations in 4 questions was completed by the HCPs.

\section{Results}

A total of 15 patients were seen by two geriatricians with Tele-SOC. The demographics of the patients are shown in Table 1. Their age, gender and race were typical of a geriatric population. All patients had their issues addressed and managed during the Tele-SOC consultations and none of the patients required an earlier physical review.

All patients needed caregivers to set up the video conferencing application to participate in the Tele-SOC consultations. ${ }^{10}$ Majority of the caregivers were children of the patients. There was no technical issue encountered for all patients. The duration of the teleconsultation ranged from 7 minutes to 89 minutes, with an average time of 39.4 minutes, which was slightly longer compared to a typical clinic slot of 30 minutes.

A total of 19 feedback were collected, 13 from patients or caregivers and 6 from HCPs (Table 2). Two patients declined to participate in the feedback. Their reasons for declining were not sought.

\section{Discussion}

In this current paper, communication was deemed to be satisfactory by majority of the patients and caregivers. The instructions in setting up the video conferencing application were perceived as easy to follow by patients and caregivers. It was pertinent to consider the usability of these systems within the geriatric population, which might be impacted by age-related factors such as vision, hearing, cognition and motor skills, amongst others. ${ }^{11}$ Our study overcame this by having caregivers as the end users, thereby increasing patients' confidence in navigating the system. ${ }^{11}$

While majority of them agreed that they were satisfied with the teleconsultations and would be willing to use it again or recommend it to others, only $38.5 \%$ of the patients and caregivers would prefer teleconsultation to physical consultation. While the use of telemedicine had been well accepted by patients and their caregivers, a lack of physical contact between the HCP and patient remained a factor for patients and caregivers to prefer physical consultations where face-to-face communication and physical examinations could be performed. ${ }^{12,13}$ This however, could be perceived as an imperative benefit of telemedicine as a means to reduce disease transmission during a pandemic. Among the feedback, two patients/caregivers preferred to be reviewed by their regular geriatrician instead of the primary attending geriatrician who conducted the Tele-SOC. However,
Table 1. Demographics of Patients

\begin{tabular}{ll}
\hline Characteristic & $\begin{array}{l}\text { Patients, } \\
\mathbf{N}=\mathbf{1 5} \\
\mathbf{n}(\mathbf{r a n g e} \text { or \%) }\end{array}$ \\
\hline Age (range) & $84(72-91)$ \\
\hline Gender & \\
\hline Female & $8(53)$ \\
\hline Male & $7(47)$ \\
\hline Race & $12(80)$ \\
\hline Chinese & $2(13)$ \\
\hline Malay & $1(7)$ \\
\hline Indian & $1(7)$ \\
\hline Main Diagnoses & $10(66)$ \\
\hline BPSD & $1(7)$ \\
\hline Skin conditions & $7(47)$ \\
\hline Chronic Disease management & $3(20)$ \\
\hline Insomnia & $4(26)$ \\
\hline Caregivers Present & \\
\hline Child & \\
\hline Child-in-law & \\
\hline Grandchild & \\
\hline Spouse & \\
\hline
\end{tabular}

given the unprecedented circumstances of a pandemic outbreak, patients and caregivers generally appreciated the convenience of teleconsultation and minimisation of exposure to COVID-19 disease.

The feedback from the HCPs revealed that communication was deemed satisfactory and instructions were easy to follow. HCPs felt that teleconsultations were generally time efficient, however, there were two who felt otherwise. While there was no elaboration on their views, they could be due to the time required to explain and guide the patients and caregivers through the steps prior to their initial teleconsultation appointments. Notably, compared to patients and caregivers, $66.7 \%$ of the HCPs preferred not to use teleconsultation. One of the reasons could be reduced reliability of information provided due to a lack of physical examination. ${ }^{14}$ In their open-ended feedback, the overall sentiment was that a lack of physical examination might not provide them with accurate clinical findings, hence, leading to a less than ideal management. Given the nature of telemedicine, consultations that require 


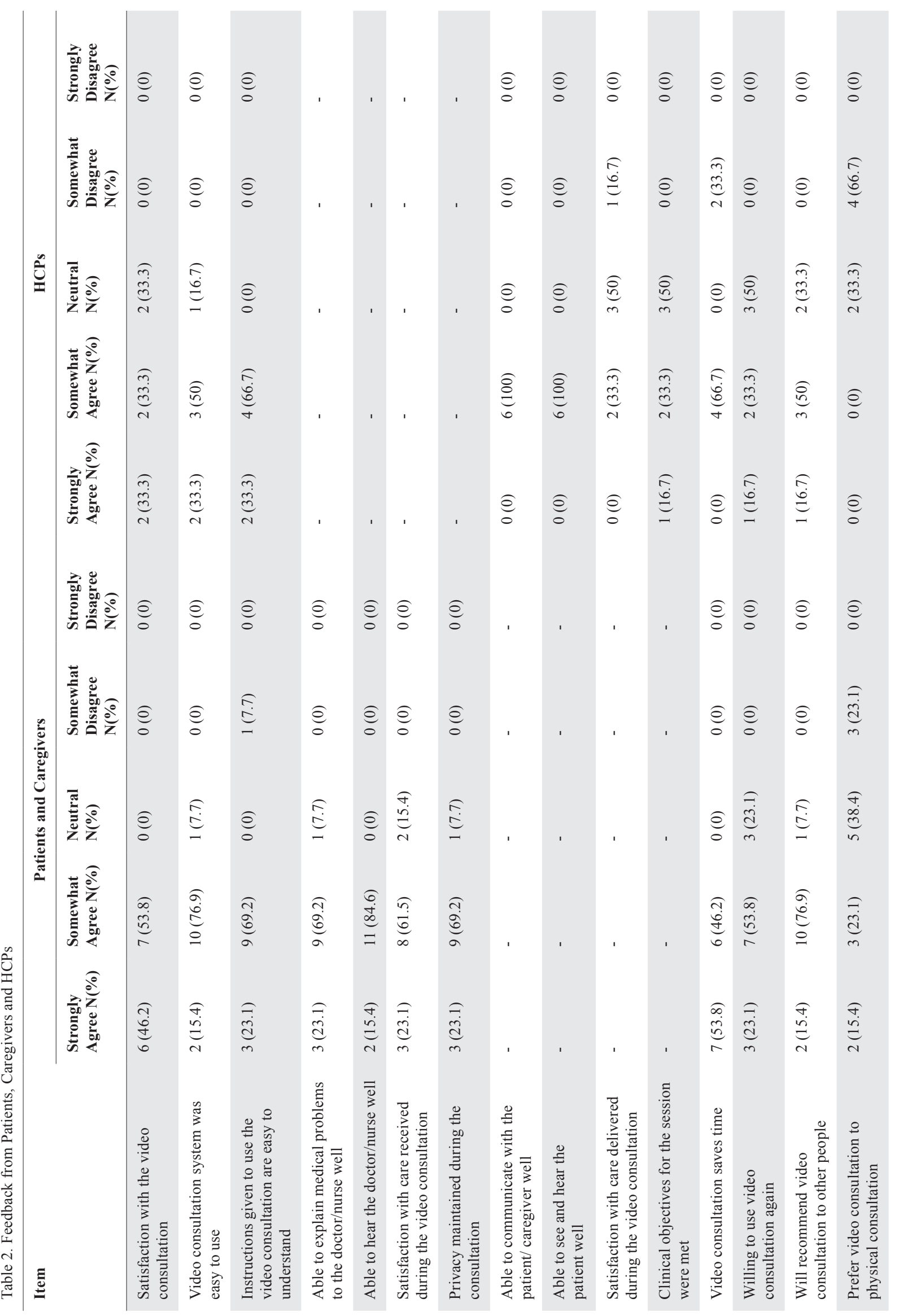


physical examinations (e.g. auscultation, palpation) and diagnostics examinations may be difficult to perform remotely, thereby, limiting the conditions which can be managed over teleconsultation. ${ }^{8}$ This prompts for careful patient selection based on criteria such as age, cognitive impairment, geri-psychiatric issues and specific chronic health conditions. ${ }^{11}$ Furthermore, telemedicine reduced the traditional face-to-face doctor-patient interaction, which might be negatively perceived by HCPs and in turn, impacting on their receptiveness to telemedicine. ${ }^{14}$ On the whole, however, HCPs expressed that teleconsultation served as a good alternative to physical consultation during a national outbreak.

As aged care services are rapidly established in Singapore, it is imperative to include telemedicine into the existing comprehensive framework of services in order to meet the evolving needs of our ageing population. ${ }^{15}$ With Singapore moving into Phase 2 of reopening on 19th June $2020,{ }^{3}$ it is essential to review and restructure delivery of health services by leveraging on technology to facilitate optimal and safe care delivery while minimising the risk of direct human-tohuman transmission, especially in a vulnerable geriatric population. ${ }^{9}$ The results of this proof of concept study demonstrated that Tele-SOC had a role in managing clinic patients in a national crisis.

Our study comprised of a small sample size that might not be generalisable to other specialist outpatient clinics in other countries. Furthermore, a lack of prior studies on telemedicine for specialist outpatient clinics might limit the scope of our discussion and comparison.

\section{Conclusion}

Our study showed that Tele-SOC provided a good alternative to physical consultations for selected groups of the geriatric population in order to minimise their exposure to virus transmission. As the current paper is a preliminary study, more research should be undertaken to determine the acceptability, safety, feasibility, reliability and implementation of telemedicine for outpatient geriatric care in Singapore, fostering user acceptance of telemedicine. Studies with larger sample sizes and a focus on geriatric population are warranted to investigate the benefits and barriers so that usability issues are addressed for effective use by the elderly.

\section{REFERENCES}

1. World Health Organization. Timeline of WHO's response to COVID-19. Available at: https://www.who.int/news-room/detail/2906-2020-covidtimeline. Accessed on 30 June 2020.
2. Ministry of Health Singapore. Continuation of essential healthcare services during period of heightened safe distancing measures. Available at: https:/www.moh.gov.sg/news-highlights/details/ continuation-of-essential-healthcare-services-during-period-ofheightened-safe-distancing-measures. Accessed on 30 June 2020.

3. Ministry of Health Singapore. Updates on COVID-19 (Coronavirus disease 2019) local situation. Available at: https://www.moh.gov.sg/ covid-19. Accessed on 30 June 2020.

4. Feast A, Moniz-Cook E, Stoner C, Charlesworth G, Orrell M. A systematic review of the relationship between behavioural and psychological symptoms (BPSD) and caregiver well-being. Int Psychogeriatr 2016;28:1761-74.

5. Foo CL, Chan KC, Goh HK, Seow E. Profiling acute presenting symptoms of geriatric patients attending an urban hospital emergency department. Ann Acad Med Singap 2009;38:515-520.

6. Jones BN. Telepsychiatry and geriatric care. Curr Psychiatry Rep 2001;3:29-36.

7. Zhai Y, Wang Y, Zhang M, Gittell JH, Jiang S, Chen B, et al. From isolation to coordination: how can telemedicine help combat the COVID-19 outbreak? medRxiv. 2020 Feb 23;Preprint.

8. Smith AC, Thomas E, Snoswell CL, Haydon H, Mehrotra A, Clemensen J, et al. Telehealth for global emergencies: implications for coronavirus disease 2019 (COVID-19). J Telemed Telecare 2020;26:309-313.

9. Hse LY, Chia PY, Lim JFY. The novel coronavirus (SARS-CoV-2) pandemic. Ann Acad Med Singap 2020 Mar;49:105-107.

10. Zoom. Available at: https://zoom.us/healthcare. Accessed on 30 June 2020.

11. Narasimha S, Agnisarman S, Welch B, Nair A. Designing telemedicine systems for geriatric patients: a review of the usability studies. Telemed J E Health 2017;23:459-472.

12. Buckley KM, Tran BQ, Prandoni CM. Receptiveness, use and acceptance of telehealth by caregivers of stroke patients in the home. Online J Issues Nurs 2004;9:9.

13. Low JA, Beins G, Lee KK, Koh E. Last moments of life: can telemedicine play a role? Palliat Support Care 2013;11:353-5.

14. Wernhart A, Gahbauer S, Haluza D. eHealth and telemedicine: practices and beliefs among healthcare professionals and medical students at a medical university. PLoS One 2019; 14:e0213067.

15. Sitoh YY. Aged care services in Singapore: an overview. Ann Acad Med Singap 2003;32:717-722.

Laurence LC Tan, ${ }^{1} M B B S$,

Vidhya Dharshini Pillay, ${ }^{1} M B B C h B A O, L R C P \& S I, G D F M$, Joanne WK Chia, ${ }^{2} B N, M P H$,

Angeline ST $\underline{\text { Seah, }}{ }^{1}$ MBBS, Dip Pall Med, FRCP,

Chun Mei Liu, ${ }^{3} B N$, Yujun Lim, ${ }^{2}$ BSpPath,

James YH Low, ${ }^{1} M B B S, M R C P, F R C P$

\footnotetext{
${ }^{1}$ Geriatric Medicine, Khoo Teck Puat Hospital, Singapore

${ }^{2}$ GeriCare, Khoo Teck Puat Hospital, Singapore

${ }^{3}$ Geriatric Outpatient Clinic, Khoo Teck Puat Hospital, Singapore
}

Address for Correspondence: Dr Laurence Tan, Geriatric Medicine, Khoo Teck Puat Hospital, 90 Yishun Central, Singapore 768828

Email: tan.laurence.lc@ktph.com.sg 